\title{
DISECTOR PROGRAM FOR UNBIASED ESTIMATION OF PARTICLE NUMBER, NUMERICAL DENSITY AND MEAN VOLUME
}

\author{
ZOLTÁN TOMORI ${ }^{1}$, IVAN KREKULE ${ }^{2}$ AND LUCIE KUBÍNOVÁ ${ }^{2}$ \\ ${ }^{1}$ Institute of Experimental Physics, Slovak Academy of Sciences, Watsonova 47, 04353 Košice, Slovak \\ Republic, ${ }^{2}$ Institute of Physiology, Academy of Sciences of the Czech Republic, Vídeňská 1083, 14220 Prague \\ 4, Czech Republic \\ e-mail: kubinova@biomed.cas.cz \\ (Accepted June 8, 2001)
}

\begin{abstract}
A DISECTOR program is presented, offering the possibility to count particles by the disector or unbiased sampling brick principles as well as to apply the point-counting method needed for estimation of the particle volume density or mean particle volume. Three modes of counting, two semi-automatic and one automatic, are offered, allowing the user to choose the one most suitable for his image data. In a semi-automatic regime, the user marks and counts individual particles by a mouse during browsing through the stack of images. In the algorithm working in an automated mode, the role of a human operator is suppressed, assuming that segmented objects are available in individual levels. The settings of the point grid and 3-D probe can be tailored for each application. The DISECTOR program applications are shown on the examples of the estimation of the number and numerical density of mesophyll cells in a Norway spruce needle and the mean volume of tubular cells in a chick embryonic kidney.
\end{abstract}

Keywords: confocal microscopy, disector, point counting, stereology, unbiased sampling brick.

\section{INTRODUCTION}

For unbiased estimation of the number of threedimensional (3-D) particles (e.g. cells) it is necessary to use such counting rules that guarantee the particles to be counted with a uniform probability in 3-D space, irrespective of their size and shape. There is no general rule ensuring unbiased sampling of 3-D particles if only a single 2-D section of the particle population is available. The reason is obvious: The probability that the section is hitting the particle is proportional to its height (in the direction perpendicular to the section) which means that higher particles are more likely to be intersected by the section (see Fig. 1). Thus, it is not correct to count particles of different heights by counting their profiles seen in a section, and so, e.g., still often used procedure of counting cells by counting their profiles in a histological section is necessarily biased.

A simple and straightforward solution of unbiased counting of particles was found by introducing the counting rules exploiting 3-D probes, namely the disector (Sterio, 1984), optical disector (Gundersen, 1986, West and Gundersen, 1990) and unbiased sampling brick (Howard et al., 1985). In practice, these counting rules are especially well applicable if series of optical sections of the particle population are available, e.g. by focusing through a thicker physical section in an optical microscope (whether using a conventional or a confocal microscope), browsing through sections obtained by MRI, etc.

The unbiased rules for counting 3-D particles (e.g. cells) sample particles regardless their size and shape under the condition that the particles can be identified unambiguously in the examined thick section. The 3-D probe is represented by a parallelepiped that is uniform randomly positioned in the studied reference space (e.g. in an organ). The unbiased rules are based on counting only those particles that are not intersected by the exclusion planes of the 3-D probe and also lie at least partially inside the probe (Fig. 1). In practice, we are browsing through serial optical sections (i.e. rectangles) of the probe, i.e. a stack of images starting from the reference (ground) plane and advancing up to its (exclusion) look-up plane. The particles are counted if their profiles fulfill the above conditions, i.e. they are seen inside the probe but do not intersect the exclusion planes. The distance between the consecutive planes in the stack has to be smaller than the minimum particle height: this can be always ensured by decreasing the step between the planes (Fig. 1b). The possible overlapping of optical sections (if the 
step is smaller than their thickness) has no affect on the counting procedure as no particle can be counted more than once in this case.

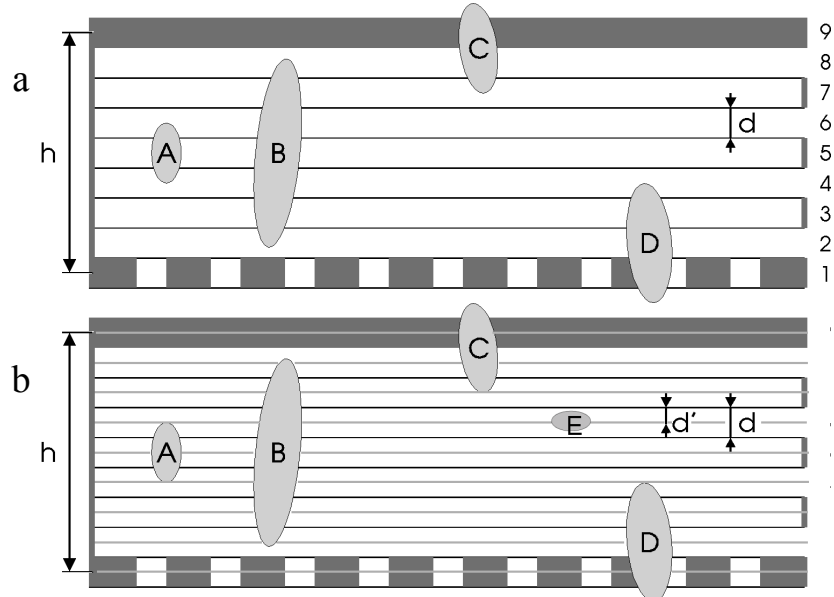

Fig. 1. The three-dimensional probe for unbiased counting of particles (side view) from a stack of optical sections. The reference plane is represented by the lowest optical section, No.1 in (a), the look-up plane by the upmost, gray optical section, No. 9 in (a). We count only those particles that are not intersected by the look-up, exclusion plane and also lie at least partially inside the probe. In (a), three particles $(A, B$, and $D)$ are counted, i.e. $Q^{-}($par $)=3$. In (b), also the particle $E$ is counted, i.e. $Q^{-}$( par $)=4$. The height of the probe is denoted by $h$. In (a), the optical section thickness is equal to the distance $d$ between the sections and there are 9 sections in the probe. In (b), the height of the particle $E$ is smaller than the section thickness, therefore a smaller step $d^{\prime}\left(d^{\prime}=d / 2\right)$ between the optical sections is taken which means there are 17 sections in the probe. The overlapping of optical sections has no affect on the counting procedure.

In optical disector (Gundersen, 1986; Gundersen et al., 1988; West and Gundersen, 1990), the particle is sampled if both following conditions are met: (i) The particle top profile is lying inside the thick section within the physical slice. (ii) This top profile is sampled by the unbiased sampling frame placed into the reference plane moving through the thick section. In practice, the thick section is simply focused through and particle profiles that come into sharp focus are sampled by the sampling frame. The optical disector is especially suitable for counting human or animal cells by counting their nuclei using a conventional transmission microscope (see. e.g. West and Gundersen, 1990) because such nuclei are usually convex, nearly spherical in shape and only their central optical sections come into a sharp focus. For counting particles of more complicated, even non-convex shapes, or objects with boundaries seen in a sharp focus within their entire depth (e.g. plant cells), it can be recommended to use rather the unbiased sampling brick rule and possibly a confocal microscope.

In the unbiased sampling brick rule (Howard et $a l ., 1985)$, the particle is sampled if it is lying at least partly in the sampling brick and at the same time it is not intersected by the exclusion planes. In a microscope, the particles lying within the thick slice are focused through and checked if any of the particle profiles is intersected by the exclusion line of the sampling frame displayed on the optical sections. If applying the unbiased sampling brick rule in a transmission microscope, there is often problem to see the caps of cells sharply enough. In confocal microscopy, usually clear and sharp images of the cell profiles can be observed and so the method is well applicable.

In both methods, we are browsing through serial sections of the 3-D probe, i.e. a stack of images starting from the reference (ground) plane and advancing up to its look-up (exclusion) plane. If the positions of the sampling frames are uniform over all possible positions in the reference space and a point grid of $p$ points is placed in the frame, the number of particles per unit volume of reference space $\left(N_{V}(\right.$ par $\left.)\right)$ can be estimated by a practically unbiased estimator:

$$
\operatorname{est}_{V}(\text { par })=\frac{\sum_{i=1}^{n} Q_{i}^{-}(\text {par })}{\sum_{i=1}^{n} P_{i}(\text { ref })} \cdot \frac{p}{a \cdot h}
$$

where $n$ is the number of disectors (sampling bricks), $Q_{i}^{-}$(par) $(i=1, \ldots, n)$ is the number of particles sampled by the $i$-th disector ( $i$-th sampling brick), $P_{i}$ (ref) $(i=1, \ldots, n)$ is the number of points of the $p$ point grid in the $i$-th frame hitting the reference space, $a$ is the actual area of the frame, and $h$ denotes the height of the disector (sampling brick), see Fig.1. If the volume $V(r e f)$ of the reference space (e.g. an organ) is known, the total number of particles in the reference space $(N($ par $))$ can be estimated by est $N_{V}($ par $)$ x $V($ ref $)$. Further, the mean particle volume can be estimated by a similar measurement using particle counting: 


$$
e s t \bar{v}_{N}(p a r)=\frac{\sum_{i=1}^{n} P_{i}(\text { par })}{\sum_{i=1}^{n} Q_{i}^{-}(\text {par })} \cdot \frac{a \cdot h}{p}
$$

where $P_{i}$ (par) $(i=1, \ldots, n)$ is the number of points of the $p$-point grid in the $i$-th frame hitting the particle profiles.

The disector principle can be implemented manually by focusing through the specimen directly under a microscope by naked eyes and counting particles sampled by the test frame, which represents a section of the probe. The test frame is usually engraved on a glass plate or drawn on a transparent sheet placed into the optical pathway. However, modern stereology requires both the selection of a proper method and the corresponding software. A STESYS program module for the generation of stereological test systems was published not long ago (Tomori et al., 2000; demo version can be downloaded from http://www.saske.sk/ tomori). A special DISECTOR program is introduced in the present study. It offers the possibility to count particles by both above mentioned unbiased principles as well as to apply the point-counting method needed for estimation of the particle volume density or mean particle volume.

\section{PROGRAM DESCRIPTION}

The DISECTOR program was developed in two versions: The first one represents a plug-in module of free image processing program ImageTool which was developed at the University of Texas, Health Care Center, San Antonio and which is available via Internet. The second version is independent of any host program, it is easy to use, but its general image processing capabilities are limited compared with the ImageTool version. Both versions require PC supplied with MS Windows 95/NT 4.0 operating system (or higher).

The DISECTOR program was designed with the aim to make the particle counting procedure efficient and comfortable. It simulates focusing through the specimen by using the slider control which allows browsing through the stack of images (Fig. 2). The test frame is superimposed on the image. Three modes of counting, two semi-automatic and one automatic, are offered, allowing the user to choose the one most suitable for his image data. In a semi-automatic regime, the user marks and counts individual particles by a mouse. In the algorithm working in an automated mode, the role of a human operator is suppressed, assuming that segmented objects are available in individual levels. The semi-automatic implementation is suitable, whenever particles of interest must be segmented in an interactive regime. Further, a pointcounting mode is available so that the reference volume or volume of particles can be estimated (see Eq. 1,2). The input data are represented by the image stack and the output is represented by the spreadsheet form with results of the measurement.

The analysis of an image stack opened in the ImageTool environment proceeds in two steps:

1. A test point grid is applied to one of the images and points falling into the reference space (Eq. 1) or particle profiles (Eq. 2) are counted. The result is stored into the Results window.

2. The particles sampled by an unbiased 3-D probe are counted: During browsing through the stack of images, particle profiles are marked by a mouse in each level within the probe. The number of sampled, so-called GOOD 3-D particles, giving the value of $Q_{i}^{-}($par $)$(Eq. 1, 2), is stored into the Results window (or file).

The settings of the point grid and 3-D probe can be tailored for each application. 


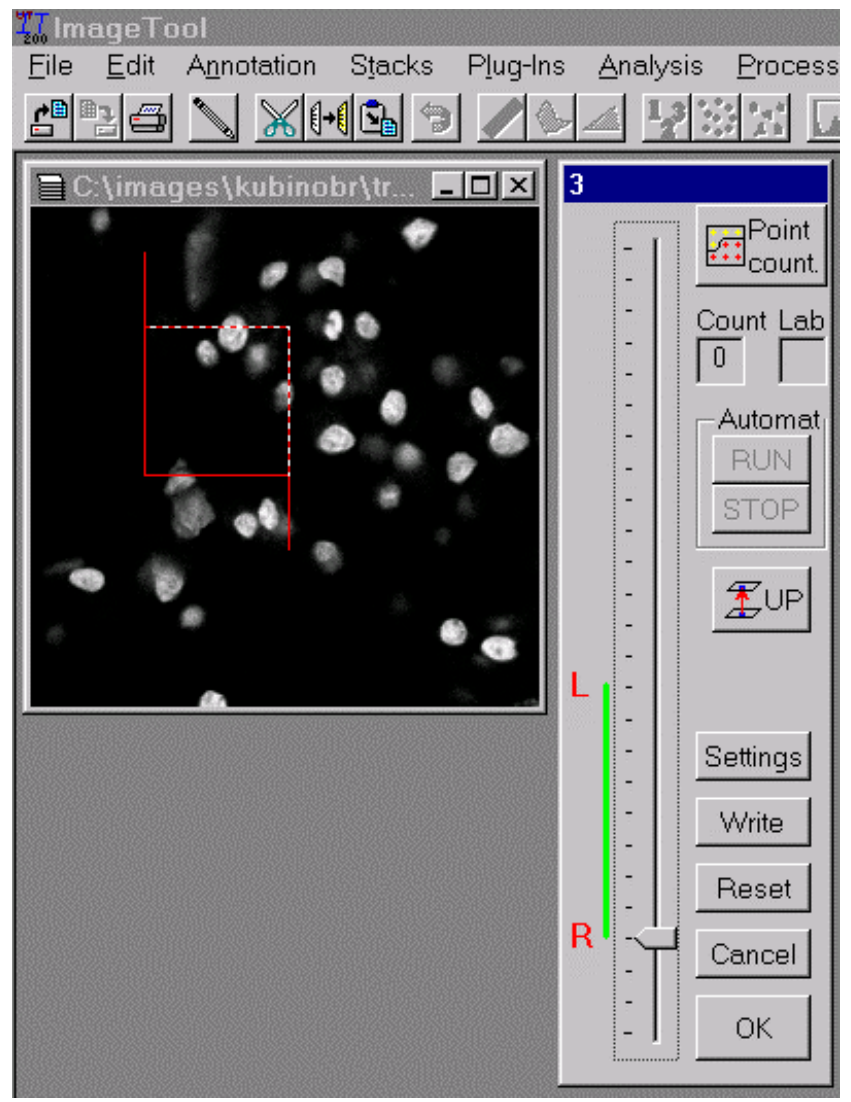

Fig. 2. The MAIN DIALOG window of the DISECTOR program containing slider for browsing through the levels of the stack. The number of the level position is displayed in the window heading, i.e. the level No .3 is shown here.

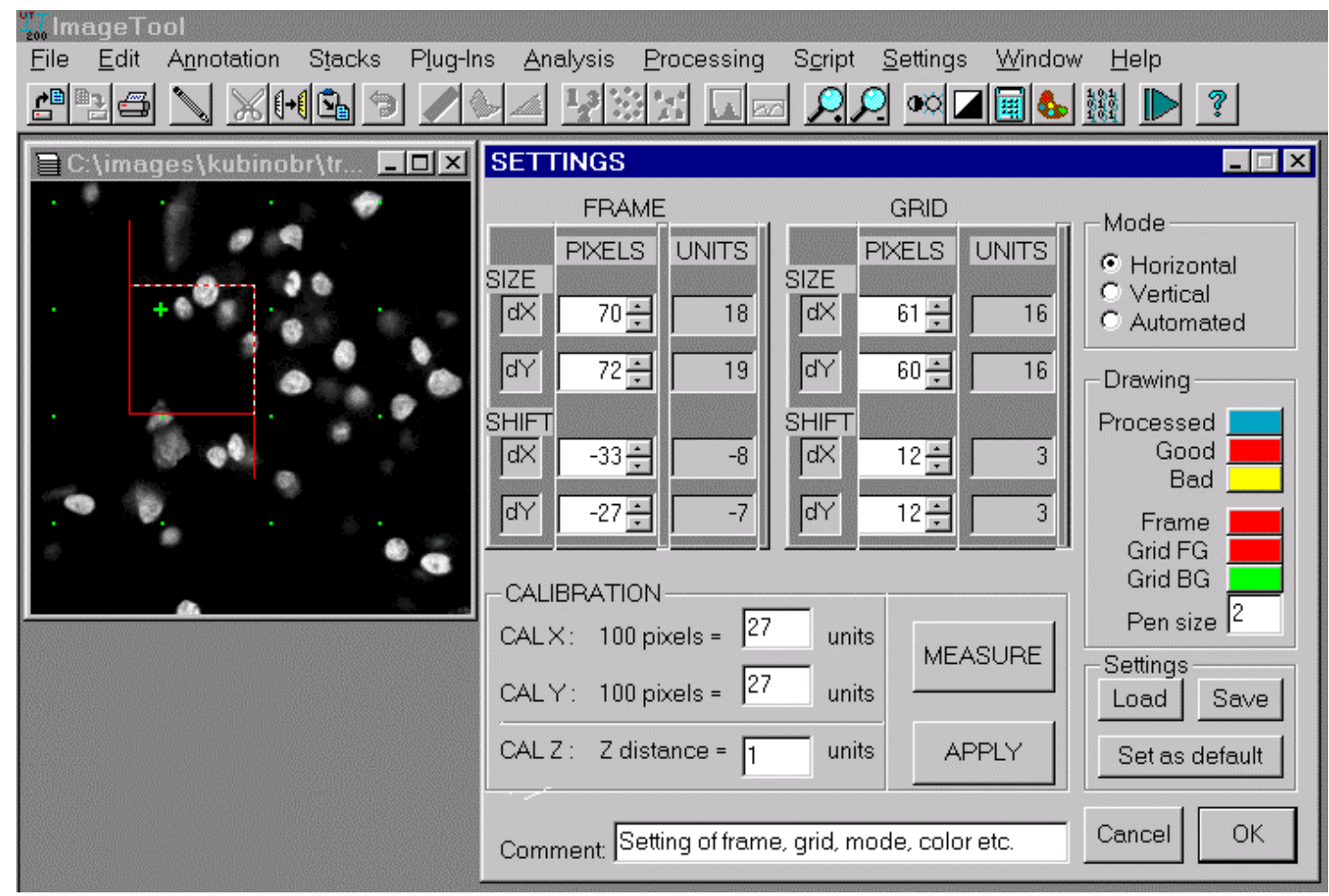

Fig. 3. SETTINGS dialog for editing and modification of all settings. 


\section{Settings}

All parameters characterizing displayed features can be set or modified in the SETTINGS dialog (Fig. 3 ). The settings can be changed and saved according to the needs of each type of the measurement. It is possible to set the calibration constants, the color of all drawn graphics (test frame, test points, marks, etc.), the size and position of the unbiased sampling frame, the distance between neighboring test points of the point grid and its position in $\mathrm{x}$ - and $\mathrm{y}$ directions, a short comment can be included and one of the three counting modes selected.

\section{Particle counting}

After the proper parameters are set, the program is controlled from the MAIN DIALOG (Fig. 2). It supports the process of counting particles sampled by the 3-D probe given by the following planes: the reference plane, the look-up plane and the four planes delimiting the unbiased counting frame in each stack level.

The main control tool is a vertical slider allowing to browse through the stack of images opened in ImageTool before starting up DISECTOR. The "active" range (i.e. the interval between the reference (R) and lookup level (L)) corresponds to the height (h) of the disector - see Eq. 1, 2. In the default setting, the $\mathrm{R}$ level is the second one from the stack and L level is set as the last but one, in order to have some guarding space above and below the 3-D probe. However, the positions of $\mathrm{R}$ and $\mathrm{L}$ levels can be changed according to the needs of the specific application.

In the MAIN DIALOG window the process of counting particles is performed according to the mode set in the SETTINGS dialog. Three modes of counting (horizontal, vertical or automated) are offered, differing in algorithms for marking particle profiles. In all modes, particle profiles are marked in each level within the probe during browsing through the stack of images (Fig. 4). A unique label (integer number shown in Lab window) is automatically assigned to the clicked structure. 2-D structures (i.e. particle profiles) in different levels having the same label form a 3-D particle. Each clicked position is initially classified as PROCESSED (displayed as a blue dot according to the color setting shown in Fig. 3 ); during the measurement it is changed either to GOOD (red dot), or BAD (yellow dot), following the counting rule. The number of GOOD 3-D particles is displayed (Count window, see Fig. 2). In all modes the mouse clicking, drag and drop function combined with a limited number of keyboard keys can be used. The drag and drop function is simplified by the cursor shape, which changes in the vicinity of a dot. The dot can be then "caught" and "moved" to the new position. Three easy-to-remember keyboard keys are exploited: CTRL, ALT and DEL. The following commands are available:

mouse click: marks particle section in the current level as PROCESSED (blue dot);

pressing CTRL + mouse click: terminates 3-D particle in the current level, reclassifies all PROCESSED dots belonging to the given particle as GOOD and redraws them in all levels (red dot);

pressing ALT + mouse click: changes the status of 3D particle (reclassifies and redraws GOOD to BAD and vice versa);

pressing DEL + mouse click: deletes all marked dots belonging to the corresponding 3-D particle (in all levels);

pressing mouse + move: moves the "caught" point into the new position where the left mouse button is released (in the current level only).

The choice of a counting mode is dependent on the type of analyzed images. 

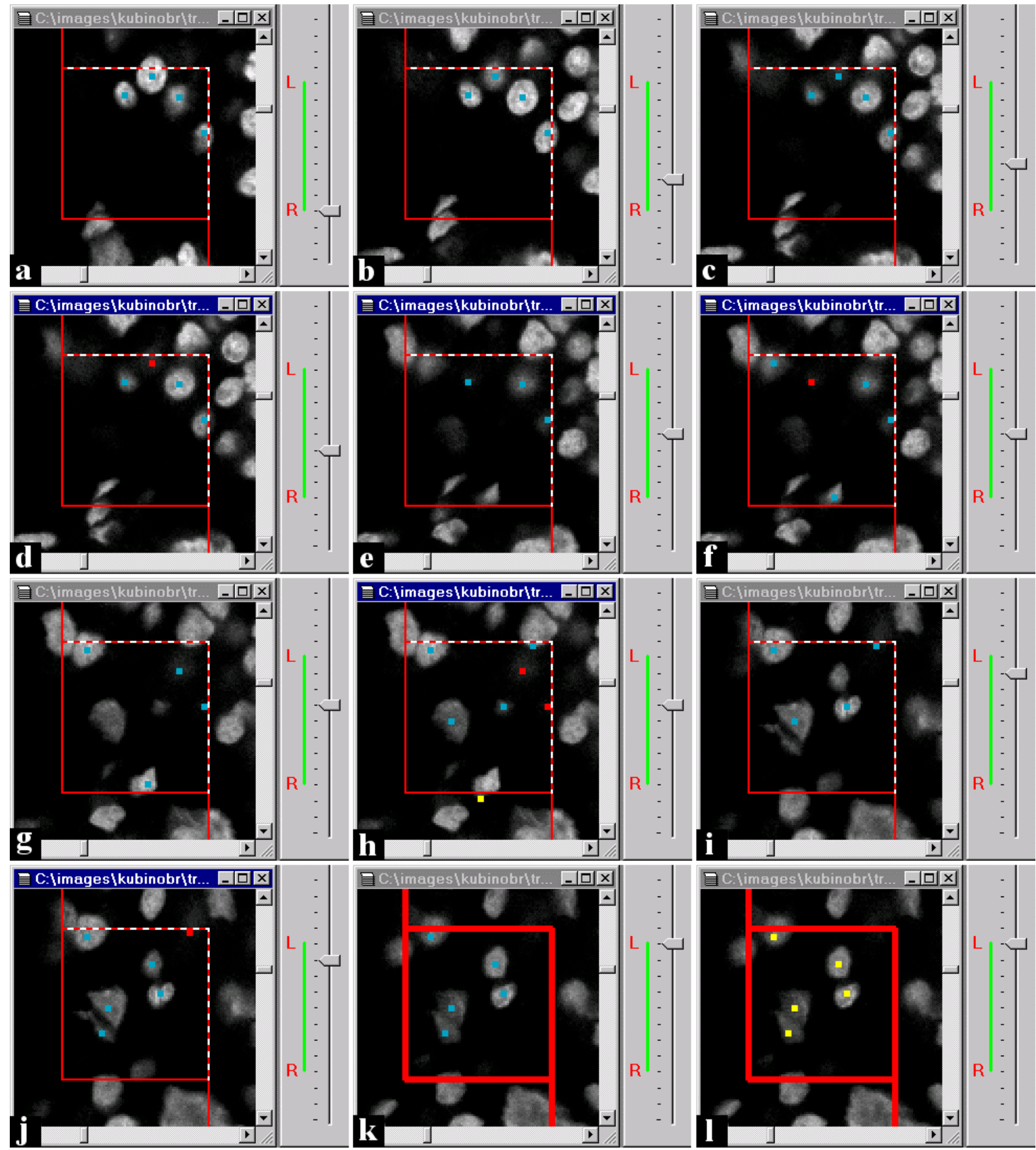

Fig. 4. Counting particles by the DISECTOR program using the horizontal mode of operation. The position of the slider thumb on the right side of each figure determines the number of image in the stack. The measurement starts from the reference level $R(a)$. Points are copied without the correction until the level $R+3(b, c)$. One particle is terminated on level $R+3(d)$. Another particle is terminated and two new particles appear on level $R+4(f)$. One particle profile touches the forbidden plane $(g)$ and the particle is classified as BAD $(h)$. Look-up level $L$ is reached $(k)$. All points remaining in the (exclusion) $L$ level are automatically classified as BAD $(l)$. Altogether five particles were counted. 


\section{Horizontal mode}

The horizontal mode (Fig. 4) is advantageous if many particles within a few levels are expected. It assumes that all particles of interest in the current level are labeled. Starting from the reference level, the particle profiles are labeled by a click; clicked points are defined as PROCESSED and displayed as blue dots (Fig. 4a). Each point is labeled by a specific label, corresponding to the order of click, which appears in the Lab window if the cursor is moved to the vicinity of the point position. After labeling all objects in the reference level, the UP button is pressed which causes the shift into the next (higher) stack level, as well as the copy of PROCESSED points from the previous level. If the position of copied points match the particle profiles (as it is in Fig. $4 \mathrm{~b}$ ) button UP can be used again, otherwise the correction should be made first, by catching and moving the point into the proper position. Fig. 4c shows the situation where the top-most blue point represents disappearing particle and therefore should be terminated. By clicking this point with the CTRL key pressed, the point is reclassified as GOOD and painted red (Fig. 4d). Another particle is terminated in Fig. 4f and two new ones appear here. In Fig.4g one particle is intersected by the forbidden plane and therefore is reclassified as BAD (by moving the corresponding dot outside the frame), i.e. painted yellow (Fig. 4h). The same strategy is applied until the look-up level is reached (Fig. 4k), where all remaining PROCESSED points became BAD (Fig. 41). The number of GOOD 3-D particles, terminating before the lookup level is reached, is displayed in the Count window. If two (or more) parts recognized first as separated particles are connected into one (nonconvex) particle during focusing, all redundant marks should be deleted so that only one label corresponds to such particle. For counting non-convex particles, the vertical mode can be more useful.

\section{Vertical mode}

The vertical mode (depth first) is convenient if a few particles appearing in many levels of the stack or particles of more complicated shape are going to be studied. The principle is similar as in the horizontal mode but each click automatically causes the movement to the next (higher) level. The multiline connecting the clicked points can be viewed as a skeleton of the tracked 3-D particle. If there is a level where the clicked point falls outside the permitted area (skeleton crosses the forbidden plane), the whole particle is labeled as BAD and repainted by a yellow color. If the tracked 3-D particle disappears in a level below the look-up plane, it should be marked as terminated by CTRL + click. Thus the particle is classified as GOOD. If the look-up plane is reached and the particle profile is still seen, the structure is BAD. After the particle is classified, the slider moves automatically back to the reference level. The number of GOOD 3-D particles is displayed in the Count window.

\section{Automated mode}

The automated mode doesn't require the human operator's assistance assuming that particles have already been segmented in the whole stack. Segmentation can be performed by some of our other ImageTool modules, e.g. SegmentTool, PSRG, PostSegmentation Thresholding, etc. (see http://www.saske.sk/ tomori). No matter which segmentation module is exploited, the output is in the same form (i.e. as a set of polygons representing contours of particles). Although this information is "hidden" in the ImageTool environment, it can be recognized by the DISECTOR module. If the segmentation is performed and the automated mode is set in the SETTINGS dialog, the RUN button in the MAIN DIALOG window should be pressed. Then the program simulates the behavior of a human operator in the horizontal mode. The criterion of continuity between profiles in two successive levels is that the profile from the upper level is overlapping with the corresponding profile projected from the lower level. If for a given level such overlapped successor doesn't exist then the corresponding 3-D particle is automatically terminated on such level (classified as GOOD in the whole stack). If the overlapped successor is crossed by a forbidden line (plane in 3D) then the corresponding 3-D particle is reclassified as BAD in the whole stack. If necessary, the markings can be changed manually using the commands described above. The current version of the algorithm does not take into account branching structures, therefore the manual assistance is necessary if the particles are non-convex.

In any of the selected modes, the output of the counting procedure should be the number of particles sampled by an unbiased 3-D probe equal to the number of particles classified as GOOD. For this purpose, it is necessary to do a final check if nonconvex particles are evaluated, i.e. to do a correction for the non-convex particles having two (or more) parts recognized as separated particles above the reference level that are connected below the reference plane (Fig. 5). This is checked by observing the levels below the reference level using the slider: If a GOOD 
3-D object is found to be connected with a BAD 3-D object under the reference plane, it should be interactively deleted leaving just a BAD mark (upper profiles in Fig. 5). If a GOOD 3-D object is found to be connected with an object in the forbidden region outside the 3-D probe (see the lower profiles in Fig. 5 ), it should be changed to BAD. If two (or more) GOOD 3-D objects are connected under the reference plane (i.e. they form just one particle), only one of them should be marked GOOD - the other GOOD object(s) should be deleted (middle profiles in Fig. 5). After checking these, in practice very rare situations, the number of counted particles is final. After pressing the Write button, it is written into the Results window having the form of a spreadsheet.
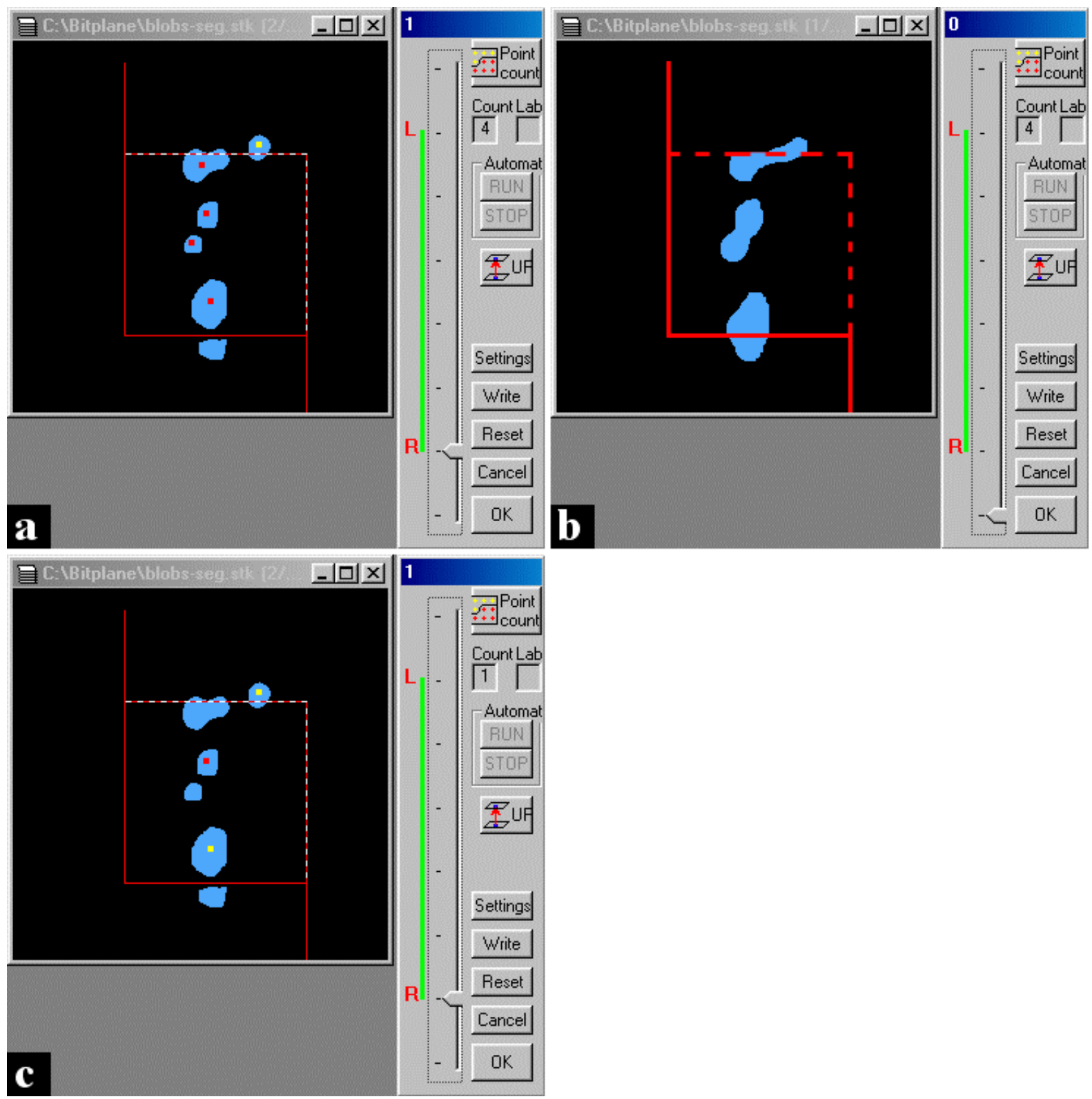

Fig. 5. The final check of counting by a 3-D probe. (a) The situation in the reference (R) level before the check. (b) The situation below the $R$ level. (c) The situation in the $R$ level after the proper corrections were made. In the middle of the sampling frame there is a GOOD particle with two GOOD profiles in the $R$ level (a) that are connected under the reference plane (b), therefore, only one of the profiles should be marked as GOOD - the second mark should be deleted (c). A GOOD 3-D object is found to be connected with a BAD 3-D object (upper profiles) under the reference plane (b), so it should be deleted leaving just a BAD mark (c). The lowest GOOD profile belongs to the small profile in the forbidden region outside the test frame (a,b), therefore it belongs to a $B A D$ particle and its mark should be changed to $B A D(c)$. 


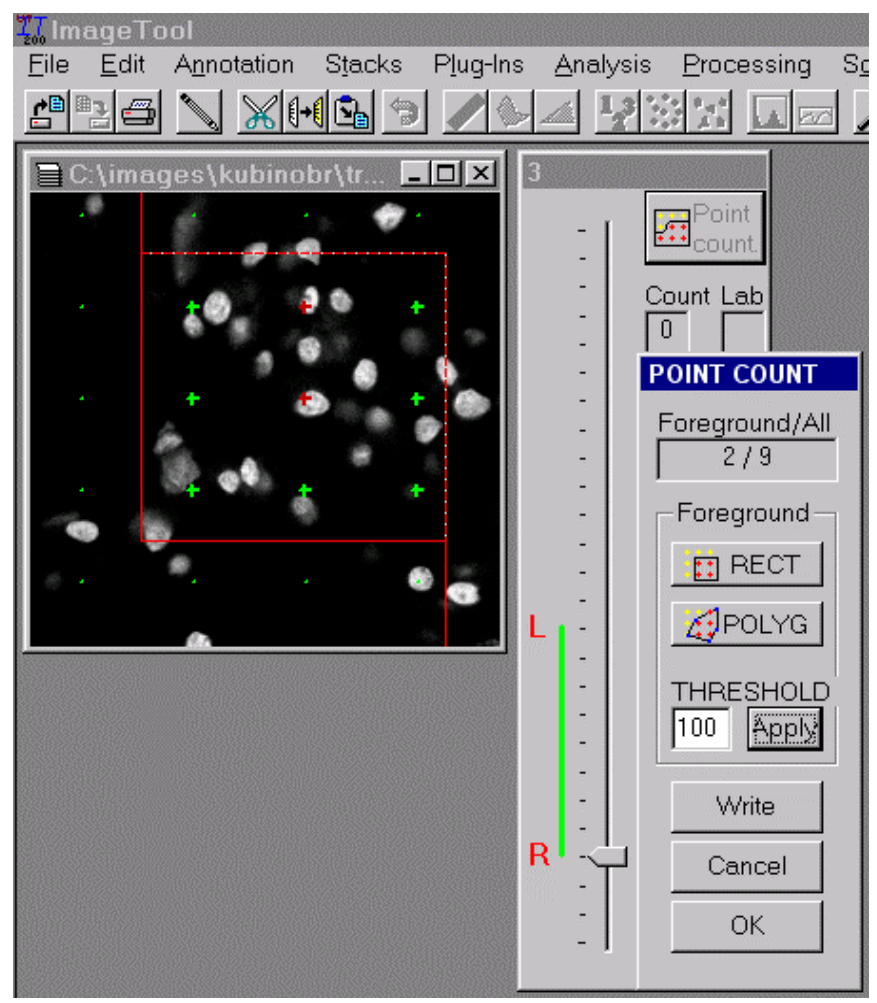

Fig. 6. Point-counting mode after applying the threshold of 100. Grid points lying in the position corresponding to the dark part of image are classified as background (green) grid points, otherwise they are the foreground (red) ones.

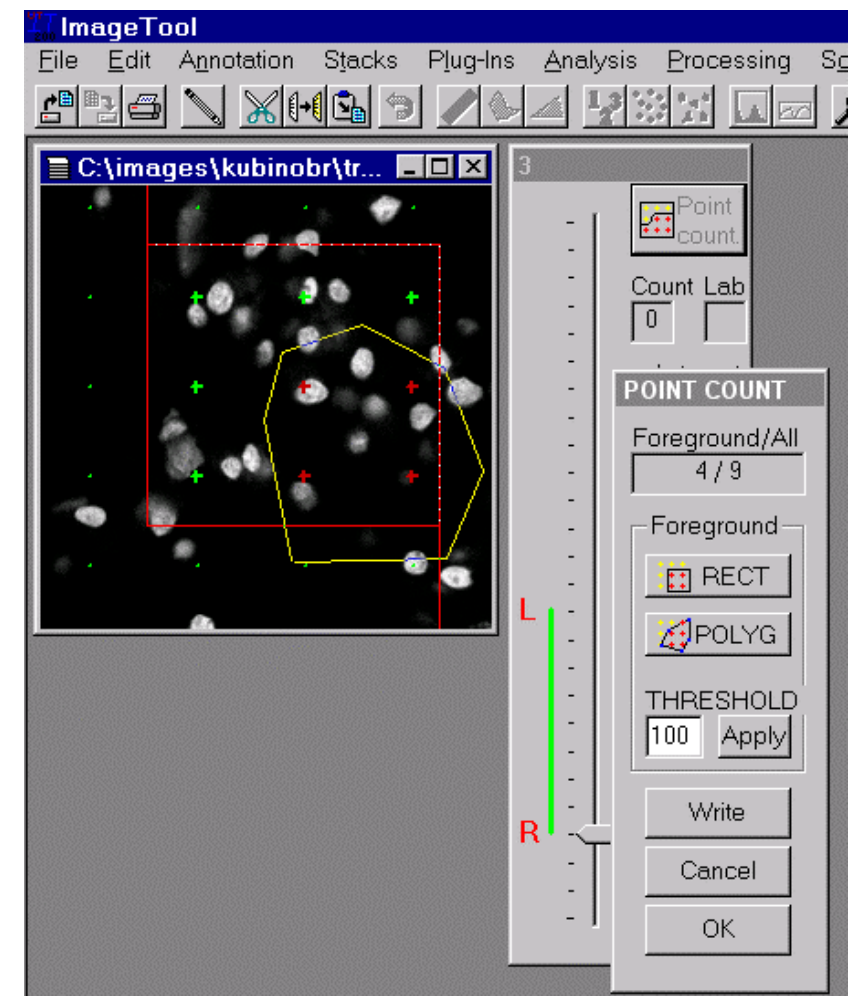

Fig. 7. Point-counting mode. Grid points inside the interactively drawn polygon are set as the foreground ones. 


\section{Point counting}

In the point-counting mode, a test point grid is applied to the selected image and points falling into the reference space (Eq. 1) or particle profiles (Eq. 2) are counted (Fig. 6, 7). At the beginning, all test points are set as background ones (green) and during the measurement the test points falling into the specified structures (i.e. reference space or particle profiles) should be changed into the foreground ones (red). There are the following possibilities how to classify points into Foreground/Background category:

a. Click near the grid point causes its re-classification and re-painting.

b. A rectangle or polygon can be defined using RECT or POLYG buttons. All grid points lying inside this geometrical structure are set as foreground and repainted (see Fig. 7 with polygon example).

c. Thresholding (Fig. 6): After pressing the Apply button, all grid points, lying "above" the pixel with gray-level intensity higher than the given THRESHOLD value, are classified as foreground ones, otherwise as background ones. This classification can be corrected manually by using method a) or b).

The actual ratio of Foreground/All grid points is displayed and can be written into the Results window.

\section{On-line mode of operation}

As one of the versions of the DISECTOR program is based on the ImageTool environment, it allows direct capturing of images using a frame grabber or a scanner. Although DISECTOR program itself has not been adapted for the full control of the frame grabber control yet (as e.g. in the C.A.S.T. Grid System, Olympus Denmark), a pseudo on-line mode of operation is available, using the built-in ImageTool support of these input devices.

The cooperation with the frame grabber is as follows: A new empty stack is created in the ImageTool environment before the DISECTOR program is started. The stack can be then filled by images from a TV camera either in a continuous or by step-by-step mode of operation. Then the DISECTOR program is started and images in the stack are analyzed. The Data Translation DT 3155 frame grabber can be recommended for the direct image capturing via the ImageTool. The adaptation for other frame grabbers would require a special driver development. Another possibility is to use a scanner supplied with the standard TWAIN interface.

\section{EXAMPLES OF APPLICATION}

\section{Number and numerical density of mesophyll cells in a Norway spruce needle}

Systematic transverse sections of the needle, about $50 \mu \mathrm{m}$ thick and $2 \mathrm{~mm}$ apart, were cut by a hand microtome and put into a droplet of distilled water between two cover glasses. They were examined by a Bio-Rad MRC600 confocal laser scanning microscope using a NIKON water immersion objective $(60 \times$, N.A. $=1.2)$ so that the refractive indices of the tissue and the immersion liquid matched. Series of 40 optical sections (with the pixel size of $0.275 \times 0.275 \mu^{2}$ ), $1 \mu \mathrm{m}$ apart, were recorded as well as the image at the $4 \mathrm{x}$ magnification showing the entire needle section. The numerical density of mesophyll cells in the needle $\left(N_{V}(\right.$ m.cell $\left.)\right)$ can be estimated using Eq. 1, by the unbiased sampling brick rule (Fig. 8) and counting test points falling within the needle. The number of all mesophyll cells in a needle $(N($ m.cell $))$ can be calculated by $N_{V}($ m.cell $) \times V($ needle $)$ if the needle volume (V(needle)) is estimated by the Cavalieri principle (see Gundersen and Jensen, 1987) applied to low-magnification images of sections. The measurement of one stack of sections took 2-4 min, i.e. one needle was evaluated in less than $100 \mathrm{~min}$.

\section{Mean volume of tubular cells in a chick embryonic kidney}

Mesonephros (embryonic kidneys) of 7-day old chick embryos were fixed in Holland solution, dehydrated in ethanol and embedded in Histoplast $\mathrm{S}$ (Kubínová et al., 1994). Sections, $20 \mu \mathrm{m}$ thick, were cut in a systematic uniform random manner and stained with haematoxylin and eosin. Stacks of 40 optical sections (with the pixel size of $0.165 \mathrm{x}$ $\left.0.165 \mu \mathrm{m}^{2}\right), 1 \mu \mathrm{m}$ apart, were recorded by a Bio-Rad MRC600 confocal laser scanning microscope using a NIKON oil immersion objective $(100 x$, N.A. $=1.4)$ The mean volume of cells of proximal tubules in the kidney can be estimated by Eq.2 where test points falling within the walls of proximal tubules are counted and the number of tubular cells is estimated by counting their nuclei using the unbiased sampling brick rule (Fig. 9). The measurement of one stack of sections took 4-5 min, i.e. one kidney was evaluated in less than three hours. Nuclei can be also counted under a conventional transmission microscope using the optical disector. 


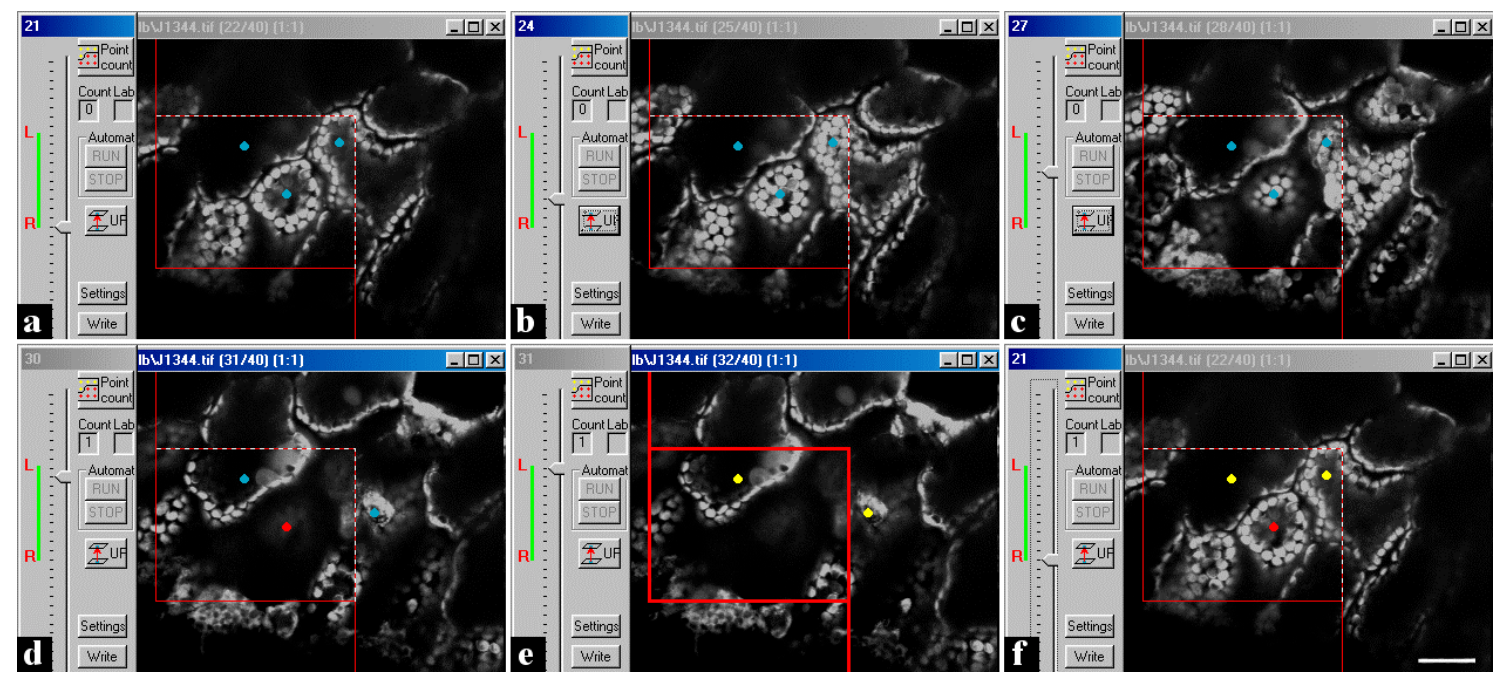

Fig. 8. Counting mesophyll cells of the Norway spruce needle by the unbiased sampling brick rule using the DISECTOR program. The reference plane was chosen to be placed in level No. 21: the situation at the beginning of the measurement is shown in (a), after the measurement in $(f)$. In (a), three cell profiles are sampled by the unbiased sampling frame. During focusing through (see level No. 24 in b, No.27 in c, No.31 in $d$ ), only the cell in the middle disappears (d), i.e. it is not intersected by the look-up, exclusion plane in (e) and it is not intersected by exclusion lines, either. Thus, this cell is sampled by the unbiased sampling brick. On the other hand, the other two cells are still seen in the exclusion plane (e) and so they are not counted (f). The height of the unbiased sampling brick is $10 \mu \mathrm{m}$ here. Scale bar $=20 \mu \mathrm{m}$.

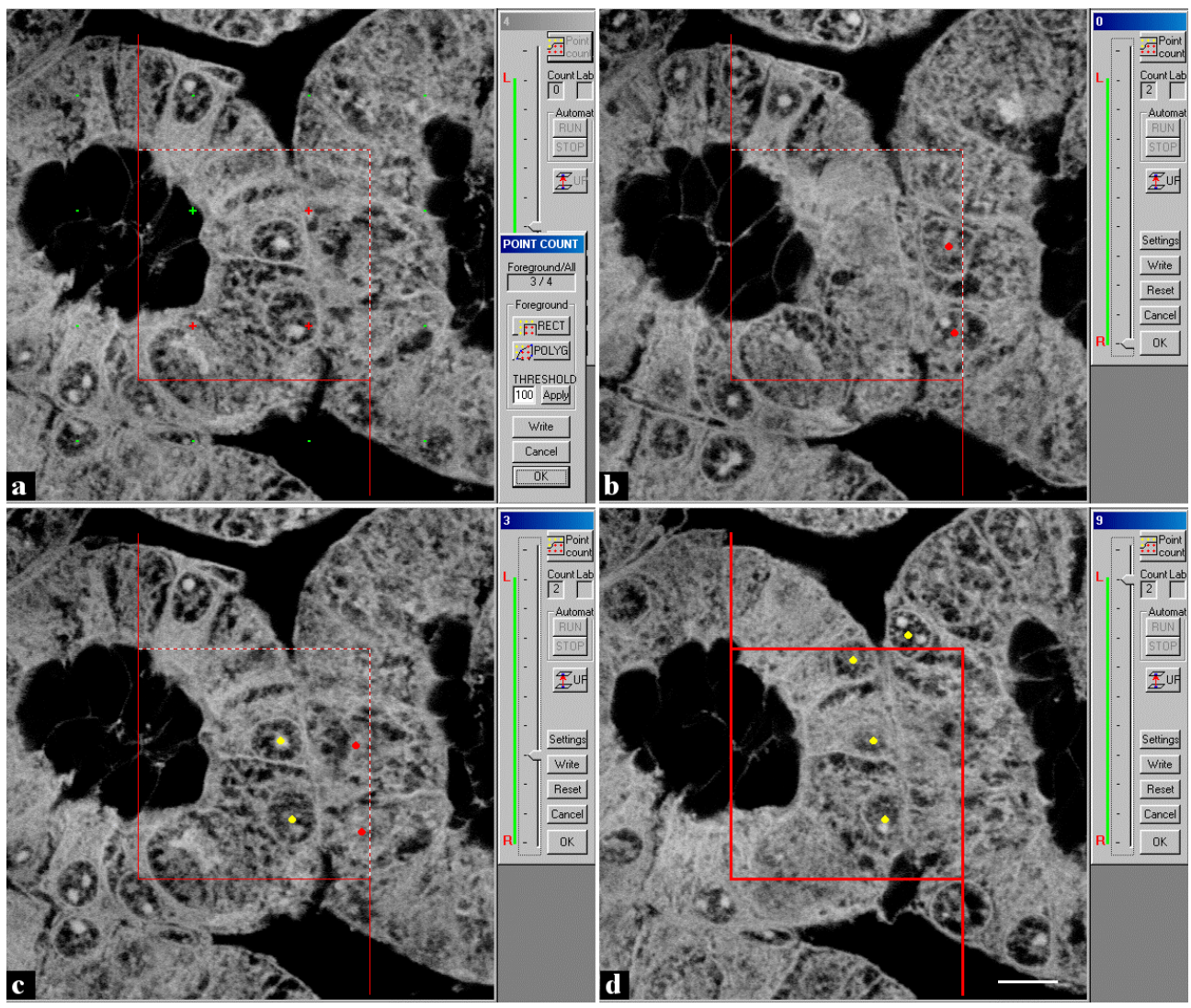

Fig. 9. Estimating the mean volume of cells of proximal tubules in the chick embryonic kidney. Test points falling within the walls of proximal tubules are counted $(a)$ and the number of tubular cells is estimated by counting their nuclei using the unbiased sampling brick rule $(b, c, d)$. The situation after the measurement is shown here: Three test points are falling within the tubular wall and two nuclei (i.e. cells) are counted. Scale bar $=10 \mu \mathrm{m}$. 


\section{DISCUSSION}

The DISECTOR program is easy to use and makes the particle counting procedure efficient and comfortable by offering different possibilities that can be tailored for each specific application. If processing of the input image data or the on-line mode of operation are needed, the ImageToolsupported version of the DISECTOR program should be used. Otherwise, the more simple and robust 'light' version can be recommended as it is not dependent on imperfections of any other program environment.

An on-line mode of operation is possible but not obligatory. Therefore, the images can be captured by one source (e.g. confocal microscope) while their processing can be performed on any $\mathrm{PC}$ with minimum hardware requirements, which makes the measurements cost-effective. Unlike many other stereological programs, three algorithms of particle counting are offered, including an automated mode. The program enables to mark the particle profiles in each level so that many particles can be counted in the same disector probe without a danger of confusion, thus larger frames (i.e. larger 3-D probes) can be easily applied. According to our practical experience, the measurement by DISECTOR is more reliable, less tiring and more than twice faster than the manual measurement based on focusing through the specimen directly under a microscope and counting particles sampled by the test frame placed into the ocular of the microscope.

The number of sampled particles and points is automatically saved into the Results spreadsheet window enabling easy evaluation of the results. Moreover, if applying the DISECTOR version running under the ImageTool system, it is possible to use special STESYS module for generating stereological test systems (Tomori et al., 2000), as well as a number of various other useful procedures for image processing, all designed with the same philosophy and type of control.

The DISECTOR program is restricted to one type of stereological measurement, unlike other, commercially available systems comprising the optical disector measurements as one of the many functions (e.g. C.A.S.T. GRID, Olympus Denmark; StereoInvestigator, MicroBrightField, Inc.; Digital Stereology, Kinetic Imaging, Ltd.; Stereologer, Systems Planning and Analysis, Inc.; BIOQUANT Stereology Toolkit, R\&M Biometrics, Inc.; for more information on contemporary stereological systems see Glaser and Glaser, 2000). Such systems can be too complex and expensive if number, numerical density or mean volume of particles, are of the main interest in the given biological experiment.

\section{ACKNOWLEDGEMENTS}

We wish to thank Dr. Zdena Zemanová (Institute of Physiology, Academy of Sciences of the Czech Republic, Prague) for providing us with the chick mesonephros specimen, Dr. Jana Albrechtová (Department of Plant Physiology, Faculty of Science, Charles University, Prague) for preparing Norway spruce needle sections, and Bitplane AG for the stack of images of Feulgen-stained cell nuclei used in Figs. $2,3,4,6,7$. The presented study was supported by the Grant Agency of the Czech Republic (grant No. 304/01/0257), the VEGA Grant Agency of the Slovak Republic (grant No. 2/1142/21) and by the Ministry of Education, Youth and Sport of the Czech Republic (KONTAKT grant No.184).

\section{REFERENCES}

Glaser JR, Glaser EM (2000). Stereology, morphometry, and mapping: the whole is greater than the sum of its parts. J Chem Neuroanat 20:115-26.

Gundersen HJG (1986). Stereology of arbitrary particles. A review of unbiased number and size estimators and the presentation of some new ones, in memory of William R. Thompson. J Microsc 143:3-45.

Gundersen HJG, Jensen EB (1987). The efficiency of systematic sampling in stereology and its prediction. $\mathrm{J}$ Microsc 147:229-63.

Gundersen HJG, Bagger P, Bendtsen TF, Evans SM, Korbo L, Marcussen N, Møller A, Nielsen K, Nyengaard JR, Pakkenberg B, Sørensen FB, Vesterby A, West MJ (1988). The new stereological tools: Disector, fractionator, nucleator and point sampled intercepts and their use in pathological research and diagnosis. APMIS 96:857-81.

Howard CV, Reid S, Baddeley A, Boyde A (1985). Unbiased estimation of particle density in the tandem scanning reflected light microscope. J Microsc 138:203-12.

Kubínová L, Zemanová Z (1994). Stereological analysis of tubular cells in normal and dilated chick mesonephric nephrons. Acta Stereol 13:95-100.

Sterio DC (1984). The unbiased estimation of number and sizes of arbitrary particles using the disector. J Microsc 134:127-36.

Tomori Z, Matis L, Karen P, Kubínová L, Krekule I (2000). STESYS2: Extended STESYS software for MS Windows. Physiol Res 49:695-701.

West MJ, Gundersen HJG (1990). Unbiased stereological estimation of the number of neurons in the human hippocampus. J Comp Neurol 296:1-22. 